\title{
Effect of Microsurgical Subinguinal Varicocele Repair on Chronic Dull Scrotal Pain in Men with Grade II-III Lesions
}

\author{
Saad Elzanatya,c Claus E. Johansen ${ }^{\mathrm{b}}$ \\ a,cDepartment of Translational Medicine, Division of Urological Research, Skåne University Hospital, Lund University, Malmö; \\ bDepartment of Radiology, Ystad Hospital, Ystad, Sweden
}

\section{Key Words}

Chronic scrotal pain $\cdot$ Microsurgery $\cdot$ Varicocele $•$

Varicocele repair

\begin{abstract}
Introduction: We aimed to evaluate the effectiveness of microsurgical subinguinal varicocele repair in patients with grade II-III lesions and chronic dull scrotal pain. Materials and Methods: A total of 29 patients with grade II-III varicocele and chronic dull scrotal pain that had a microsurgical subinguinal varicocele repair were included in the study. They were followed-up for 6-12 months including pain assessment and scrotal examination. Results: Of the 29 patients, 28 (97\%) reported complete resolution of pain with no palpable varicocele on scrotal examination. No cases of testicular atrophy or hydrocele formation were reported. Conclusion: These results indicated that microsurgical varicocele repair should be considered in patients with grade II-III lesions and chronic dull scrotal pain.
\end{abstract}

Copyright $\odot 2015$ S. Karger AG, Basel

\section{Introduction}

Varicocele defined as dilatation of the pampiniform plexus of veins surrounding the testis is observed in $10-15 \%$ of the general male population, in $20-40 \%$ of infertile men [1], and in $2-14 \%$ of men with scrotal pain $[2,3]$.

Patients with varicocele induced male infertility present with clinical varicocele (grade I, II, and III) and reduced semen quality. Most urologists agree that the primary treatment of this group of men is varicocele repair. Patients with varicocele induced scrotal pain presented with clinical varicocele and a dull scrotal pain generally after standing for a long period of time or performing heavy activity, and with an unsatisfactory response to conservative treatment. Varicocele repair to manage such a group of men, however, is still a matter of discussion.

We previously published a pilot study of microsurgical subinguinal varicocele repair from 8 patients with grade III varicocele and chronic dull scrotal pain. We reported a success rate (complete resolution of scrotal pain) in $88 \%$ of the patients [4]. The present study is a follow-up of our pilot study [4] based on a larger number of men $(n=29)$ including men with grade II and III varicoceles and chronic dull scrotal pain, and including the 8 original patients.

\section{KARGER}

Fax +4161306 1234

E-Mail karger@karger.ch

www.karger.com
(C) 2015 S. Karger AG, Basel

1015-9770/14/0094-0188\$38.00/0

Accessible online at:

www.karger.com/cur
Saad Elzanaty

Department of Translational Medicine

Division of Urological Research, Skåne University Hospital

Jan Waldenströms Gata 5

SW-20502, Malmö (Sweden)

E-Mail saad.elzanaty@med.lu.se 


\section{Material and Methods}

A total of 29 patients who had undergone microsurgical subinguinal varicocele repair because of chronic dull scrotal pain from 2012 to 2014 were included in the study. Preoperative evaluation included a detailed medical history; assessment of scrotal pain including its duration, quality (dull, dragging, and aching), and quantity using the 10 point visual analog scale for pain (VAS), (0: none, 1-3: mild, 4-6: moderate, and 7-10: severe) and a scrotal examination including measurement of testicular size using an orchidometer, assessment of the epididymis with respect to its consistency and any pain on palpation, and evaluation of the spermatic cord for the presence of a varicocele and its location (left, right, or bilateral). Varicoceles were assigned to grades I-III during examination while the patients was in a standing position according to the criteria of Lyon et al. [5] as follow: grade I: palpable only with valsalva, grade II: palpable without valsalva, and grade III: visible from a distance. Finally, the patients were examined for signs of inguinal hernia.

Patients who had other causes of scrotal pain such as testicular trauma, testicular torsion, epididymitis, prostatitis, sexually transmitted dieses, or inguinal hernia were excluded. Only patients with a clinical varicocele and chronic dull scrotal pain were included and were scheduled for microsurgical subinguinal varicocele repair. All the patients had undergone conservative treatment for pain with non-steroidal anti-inflammatory medications, scrotal elevation, and limitation of heavy activity for 3-6 months. None of the patients had any significant benefit from the conservative treatment.

Varicocele repair was performed using the microsurgical subinguinal approach according to our previous description [4]. The patients were then observed in the post-operative department and were discharged the same day.

Follow-up evaluation was done at one month [for early postoperative complications (wound infection and hematoma)], and at 6-12 months after surgery [for late postoperative complications (testicular atrophy, hydrocele formation, or persistence/recurrence of the varicocele), and assessment of scrotal pain using VAS]. The follow-up evaluation was done by one examiner. The scrotal examination was done both clinically and with a scrotal Doppler probe. Unfortunately, the main scrotal vein diameter was only recorded in 7 men with a mean value of $2.1 \mathrm{~mm}$ (range 1.2-2.7 $\mathrm{mm}$ ), and for which reason we preferred not to show this data.

The patient's response after surgery were graded as complete response (pain was absent after surgery), partial response (pain persisted but was reduced after surgery), and no response (pain remained unchanged after surgery). Surgical success was defined as absence of a palpable varicocele on scrotal examination, and a report of scores in the lowest range of the VAS ( $\leq 1$ point).

\section{Results}

The mean age of these men was 31 years (range 18-66 years). The scrotal examination revealed normal testicular size and normal epididymal findings. There was a grade II varicocele in $9(31 \%)$ patients (8 left-sided and 1 right-sided), and a grade III varicocele in $20(69 \%)$ pa-
Table 1. Descriptive statistics of the study population

\begin{tabular}{ll}
\hline Variables & Value \\
\hline $\begin{array}{l}\text { Age (years) } \\
\text { Pain }\end{array}$ & $31(18-66)$ \\
$\quad$ Pain quantity*** & \\
Varicocele grade & $4-10$ \\
I & \\
II ${ }^{* * * * *}$ & 0 \\
III & 9 \\
Varicocele laterality & 20 \\
Unilateral & \\
Left & 29 \\
Right & 28 \\
Bilateral & 1 \\
\end{tabular}

Data are mean (range) ) $^{*}$ number. ${ }^{* *}$ Was assessed using the 10 point visual analog scale for pain. ${ }^{* * *} 8$ were left-sided and 1 right-sided.

Table 2. Results of microsurgical subinguinal varicocele repair on 29 men with grade II-III lesions and chronic dull scrotal pain reported at 6-12 months after surgery.

\begin{tabular}{ll}
\hline Variables & Value, $\mathrm{n}$ \\
\hline Pain resolution (VAS): & \\
$\quad$ Complete & $28(0-1)$ \\
$\quad$ Partial & $1(4)$ \\
Post-operative complications: & \\
$\quad$ Wound infection & 0 \\
Hematoma & 2 \\
Hydrocele & 0 \\
Testicular atrophy & 0 \\
Persistence/Recurrence & 1 \\
\hline
\end{tabular}

tients (all were left-sided). None of the patients had any signs of inguinal hernia. All patients reported a dull non -radiating scrotal pain that increase in intensity with exercise. The duration of pain was of more than 6 months. The quantity of pain using the VAS ranged from 4-10 (table 1).

The mean operative time was 65 min (range 47-78 min). No intraoperative complications were reported. At the early postoperative visit, only 2 of the 29 (7.0\%) patients presented with mild haematoma which did not require surgical intervention. No other complications were reported. At the late postoperative visit, 28 of the 29 (97\%) patients reported a complete response (VAS 0-1), with no palpable varicocele on scrotal examination. Only one patient (3.0\%) reported partial scrotal pain (VAS 4). 
On scrotal examination, he still had a grade III varicocele on the operation side (left side). No evidence of testicular atrophy or hydrocele formation was found in any patient (table 2). None of the men required any type of post operative treatment.

\section{Discussion}

Our study was based on 29 patients with a grade II-III varicocele and chronic dull scrotal pain who had undergone microsurgical subinguinal varicocele repair and were followed-up for 6-12 months after surgery including assessment of scrotal pain. Of these 29 patients, $28(97 \%)$ reported a complete resolution of pain. Our results are in accordance with previous reports $[2,4,6$, 8]. We, therefore, believe that microsurgical varicocele repair should be considered in men with a grade II-III varicocele and chronic dull scrotal pain.

It has been reported that the success of the surgical treatment of painful varicoceles depends on the character of the pain. Thus, it has been observed that patients who presented with dull non-radiating scrotal pain had a significantly higher rate of success in terms of resolution of pain than those who presented with sharp scrotal pain [3]. The duration of pain was also reported to affect the success of the surgical treatment of painful varicoceles. Previous studies showed that men who presented with a long period of scrotal pain (> 3 months) had a significantly higher rate of success in terms of resolution of pain after surgery as compared to men who presented with a short period of scrotal pain $(<3$ months) [7]. In accordance, our patients presented with dull non-radiating chronic scrotal pain.

Persistence/recurrence of a varicocele is an additional factor that should be considered when evaluating the success of the surgical treatment of painful varicoceles. In this matter, microsurgical varicocele repair was reported to be associated with a significantly lower rate of persistence/recurrence than non-microsurgical varicocele repair $[9,10]$. The contribution of the gubernacular vein to postoperative persistence/recurrence is still unclear. Goldstein et al. [11] performed 33 varicocele repairs using the non-microsurgical approach without delivery of the testis, 12 varicocele repairs using $2.5 x$ loupes without delivery of the testis, and 326 varicocele repairs using the microsurgical approach with delivery of the testis and reported a persistence/recurrence rate of $9 \%, 8 \%$, and $0.6 \%$, respectively. Schiff et al. [12] performed 74 varicocele repairs using the microsurgical subinguinal ap- proach. In each of the procedures, the testis was delivered and the gubernaculum was examined for the presence of any varicose veins between the gubernacular vein and the testis and any large veins were clipped and ligated. The authors reported no persistence/recurrence during the follow-up period which was extended to a mean of 10 months. These results suggesting that deliver of the testis and examination of the gubernacular vein reduces the risk of persistence/recurrence. The patients included in our study underwent varicoceles repair using the microsurgical subinguinal approach. However, we did not deliver the testis out of the scrotum nor did we ligate the gubernacular vein, and persistence/recurrence was found in only one patient.

The resolution of scrotal pain after surgery could be attributed to the reduction of the pressure in the testicular venous drainage system, thus enabling normal oxygen flow to the testicles with correction of hypoxia in the testicular tissue caused by hydrostatic pressure resulting from the destruction of the one-way valves in the internal spermatic veins. In addition, resection of some of the spermatic nerves during surgery is another contributing factor for pain resolution.

With the assistance of the operating microscope and the Doppler probe, we were able to identify and preserve the testicular artery and the testicular lymphatics. None of our patients had testicular atrophy or hydrocele one year after surgery.

Our study has some limitations. This study was based on a relatively small number of patients with a specific type of painful varicocele and with no control group. Moreover, we were not able to investigate the effect of post operative time on the outcome results since scrotal pain was evaluated only once at the late fellow up visit. However, our results are still valid and support the notion that varicocele repair should be considered for men with clinical varicoceles and chronic scrotal pain.

In conclusion, the results from this study indicated that microsurgical subinguinal varicocele repair should be considered in men with grade II-III lesions and chronic dull scrotal pain. 


\section{References}

11 Meacham RB, Townsend RR, Rademacher D, Drose JA: The incidence of varicoceles in the general population when evaluated by physical examination, gray scale sonography and color Doppler sonography. J Urol 1994; 151:1535-1538.

2 Peterson AC, Lance RS, Ruiz HE: Outcomes of varicocele ligation done for pain. J Urol 1998;159:1565-1567.

3 Al-Buheissi SZ, Patel HR, Wazait HD, Miller RA, Nathan S: Predictors of success in surgical ligation of painful varicocele. Urol Int 2007;79:33-36.

4 Elzanaty S, Johansen CE: Microsurgical varicocele repair on men with grade III lesions and chronic dull scrotal pain: a pilot study. Curr Urol 2015;8:29-31.
5 Lyon RP, Marshall S, Scott MP: Varicocele in childhood and adolescence: implication in adulthood infertility? Urology 1982;19:641644.

6 Yaman O, Ozdiler E, Anafarta K, Gö ü O: Effect of microsurgical subinguinal varicocele ligation to treat pain. Urology 2000; 55:107-108.

7 Altunoluk B, Soylemez H, Efe E, Malkoc O: Duration of preoperative scrotal pain may predict the success of microsurgical varicocelectomy. Int Braz J Urol 2010;36:55-59.

8 Kim SO, Jung H, Park K: Outcomes of microsurgical subinguinal varicocelectomy for painful varicoceles. J Androl 2012;33:872875 .
9 Cayan S, Kadioglu TC, Tefekli A, Kadioglu A, Tellaloglu S: Comparison of results and complications of high ligation surgery and microsurgical high inguinal varicocelectomy in the treatment of varicocele. Urology 2000; 55:750-754.

10 Diegidio P, Jhaveri JK, Ghannam S, Pinkhasov R, Shabsigh R, Fisch H: Review of current varicocelectomy techniques and their outcomes. BJU Int 2011;108:1157-1172.

11 Goldstein M, Gilbert BR, Dicker AP, Dwosh $\mathrm{J}$, Gnecco $\mathrm{C}$ : Microsurgical inguinal varicocelectomy with delivery of the testis: an artery and lymphatic sparing technique. J Urol 1992;148:1808-1811.

12 Schiff J, Kelly C, Goldstein M, Schlegel P, Poppas D: Managing varicoceles in children: results with microsurgical varicocelectomy. BJU Int 2005;95:399-402. 